\title{
Discours
}

Revue de linguistique, psycholinguistique et

informatique. A journal of linguistics, psycholinguistics and computational linguistics

$27 \mid 2020$

Varia

\section{L'alternance actif / passif en français : une étude statistique sur corpus écrit}

Yanis da Cunha et Anne Abeillé

URL : http://journals.openedition.org/discours/10956

DOI : $10.4000 /$ discours. 10956

ISSN : 1963-1723

Éditeur :

Laboratoire LATTICE, Presses universitaires de Caen

\section{Référence électronique}

Yanis da Cunha et Anne Abeillé, «L'alternance actif / passif en français : une étude statistique sur corpus écrit », Discours [En ligne], 27 | 2020, mis en ligne le 23 décembre 2020, consulté le 12 avril 2021. URL : http://journals.openedition.org/discours/10956 ; DOI : https://doi.org/10.4000/discours. 10956 

Revue de linguistique, psycholinguistique et informatique

\title{
L'alternance actif / passif en français: une étude statistique sur corpus écrit
}

\author{
Yanis da Cunha \\ Laboratoire de linguistique formelle \\ Université de Paris \\ Anne Abeillé \\ Laboratoire de linguistique formelle \\ Université de Paris
}

Yanis da Cunha, Anne Abeillé, «L'alternance actif/passif en français: une étude statistique sur corpus écrit», Discours [En ligne], 27 | 2020, mis en ligne le 23 décembre 2020.

URL: http://journals.openedition.org/discours/10956

Titre du numéro: Varia

Coordination: Shirley Carter-Thomas \& Frédéric Landragin

Date de réception de l'article: 03/07/2020

Date d'acceptation de l'article: 18/09/2020 



\title{
L'alternance actif / passif en français: une étude statistique sur corpus écrit
}

\author{
Yanis da Cunha \\ Laboratoire de linguistique formelle \\ Université de Paris \\ Anne Abeillé \\ Laboratoire de linguistique formelle \\ Université de Paris
}

\begin{abstract}
Nous étudions l'utilisation de la construction passive en français écrit, en mettant au jour des contraintes préférentielles. Nous avons extrait et annoté un échantillon de 500 phrases (250 actives et 250 passives) du corpus arboré du français (Abeillé et al., 2019) puis nous avons modélisé les données par régression logistique (Baayen, 2008). Si le passif court (sans complément d'agent) est plus fréquent que le passif long ( $77 \%$ des passifs sont courts), nous observons que dans près de $80 \%$ des cas de passif court, l'argument omis est récupérable en contexte. Nous montrons le rôle prépondérant de la longueur des arguments et de la structure informationnelle dans l'alternance actif/ passif. Nous montrons également que la construction passive obéit à des contraintes plus générales sur l'alignement harmonique des arguments (Bresnan et al., 2001 et 2007).
\end{abstract}

Mots clés: corpus écrit, passif, contraintes préférentielles, modélisation statistique

We study the active/passive alternation in written French to discover preference constraints. We have extracted and annotated a sample of 500 clauses (250 active and 250 passive) of the French Treebank (Abeillé et al., 2019) and modeled them with logistic regression (Baayen, 2008). Short passives (without a par-phrase) are more frequent than long passive (77\% of passives are short); in $80 \%$ of short passives, the omitted argument is present in the context. We show that argument length and information structure are important factors in active/passive alternation. We also show that the passive construction obeys more general cross-linguistic harmonic argument alignment constraints (Bresnan et al., 2001 and 2007).

Keywords: written corpus, passive, preference constraints, statistical modeling

Nous remercions les relecteurs de la revue, les participants au séminaire de linguistique expérimentale et plus particulièrement Barbara Hemforth, Juliette Thuilier et Heather Burnett pour leurs remarques et conseils, ainsi que Marie Candito pour la version du corpus annotée pour le passif. Ce travail a bénéficié partiellement d'une aide de l'État gérée par l'Agence nationale de la recherche (ANR) au titre du programme «Investissements d'avenir» portant la référence ANR-10-LABX-0083. II contribue à I'IDEX (Initiative d'excellence) portée par I'université de Paris - ANR-18-IDEX-0001. 


\section{Introduction}

De nombreuses études ont été consacrées au passif en français, d'un point de vue descriptif ou théorique (Zribi-Hertz, 1982; Leclère, 1993; Mathieu, 1993; Gaatone, 1993 et 1998, par exemple). Nous disposons en revanche de peu d'études empiriques quantitatives. L'étude d'Attal (1985), sur Candide de Voltaire, ainsi que l'étude de Hamma et al. (2017), sur le corpus ESLO («Enquêtes sociolinguistiques à Orléans»), ne comparent pas phrases passives et phrases actives et ne comportent pas de modélisation statistique. Les auteurs pointent toutefois quelques caractéristiques qualitatives du passif: le sujet du passif est souvent un pronom, tandis que le complément d'agent apporte une nouvelle information (Hamma et al., 2017). Certains passifs sans agent seraient à rapprocher des actifs dont le sujet est on (Attal, 1985). Par ailleurs, on note une première différence entre modalités: Hamma et al. (2017) rapportent $10 \%$ de passifs avec complément d'agent dans leur corpus oral, tandis qu'Attal (1985) en compte environ $28 \%$ dans son corpus écrit.

Notre étude s'inscrit dans le champ de la syntaxe expérimentale quantitative (Bresnan et al., 2007; Bresnan et Nikitina, 2009), appliquée au français (Thuilier, 2012a et b; Thuilier et al., 2014; Faghiri et Thuilier, 2018).

Dans ses études quantitatives consacrées à l'ordre des compléments postverbaux, Thuilier a mis au jour certains facteurs significatifs:

- la longueur (en nombre de mots): les compléments courts sont placés avant les compléments longs (Thuilier, 2012a);

- l'accessibilité: les compléments connus sont placés avant les compléments nouveaux (Faghiri et Thuilier, 2018);

- la sémantique du verbe: les verbes de communication sont plus enclins à présenter un ordre SP-SN (syntagme prépositionnel - syntagme nominal) (Thuilier, 2012b);

- le figement: les collocations entre verbe et SP comme mettre en valeur, mettre à disposition, etc., favorisent l'ordre SP-SN.

En revanche, certains facteurs comme le caractère animé n'étaient pas significatifs, contrairement à ce qui a été observé, selon les mêmes méthodes, dans d'autres langues comme l'anglais (Bresnan et al., 2007; Bresnan et Ford, 2010), l'allemand (Kempen et Harbusch, 2004), le grec moderne (Branigan et Feleki, 1999) ou le japonais (Tanaka et al., 2011). La pronominalité n'était pas non plus significative, mais on peut l'imputer au fait que la plupart des compléments pronominaux en français se cliticisent, ce qui les exclut de l'analyse de la complémentation postverbale.

Nous testons les mêmes facteurs que Thuilier (2012a) dans le cadre de l'alternance actif/passif, auxquels nous ajoutons :

- le genre, le nombre, la personne;

- le type syntaxique de la phrase (principale, type de subordonnée, etc.); 
- l'amorçage, annoté selon que la phrase précédente comporte une forme passive ou non;

- le figement: les collocations entre verbe et SP comme mettre en valeur, mettre à disposition, etc., favorisent l'ordre SP-SN.

Pour décrire l'alternance actif/passif, nous nommons «argument 1 (Arg1)» l'argument sujet de l'actif et «argument 2 (Arg2)», l'argument objet de l'actif. D'où la conception du passif long en [2] comme une inversion de l'ordre des arguments par rapport à l'actif en [1]. L'omission de l'argument 1 du passif long, appelé complément d'agent, est également possible, donnant le passif court en [3].

[1] [Une grenade lacrymogène $]_{\mathrm{ARG} 1}$ a blessé [un agriculteur $]_{\mathrm{ARG} 2}$.

[2] [Un agriculteur $]_{A R G 2}$ a été blessé [par une grenade lacrymogène $]_{A R G 1}$.

(FTB, 02/07/1992)

[3] [Un agriculteur $]_{\text {ARG2 }}$ a été blessé.

Plusieurs analyses ont été proposées concernant l'argument 1 du passif court. Les contrastes suivants, tirés de Creissels (2006), qui s'inspire de Jackendoff (1977) pour l'anglais, montrent que la construction passive est différente de la construction moyenne en se du français:

[4a] La porte a été ouverte volontairement.

[4b] \# La porte s'est ouverte volontairement.

[5a] \# La porte a été ouverte toute seule.

[5b] La porte s'est ouverte toute seule.

Contrairement à ce qui se passe dans la construction moyenne, dans le passif court, l'argument 1 reste implicitement présent. En effet, en [4a], l'adverbe agentif volontairement est possible. En revanche, en [4b] ce même adverbe n'est pas admis, car le moyen supprime la participation d'une entité agentive dans l'évènement. En [5], on observe le contraste inverse, car tout seul dénote l'absence de participation d'une autre entité. Un tel modifieur est bien incompatible avec le passif, puisque la construction implique la participation implicite de l'agent. Un de nos objectifs est de tester si l'argument omis au passif court est effectivement récupérable en contexte dans un corpus, et s'il a plutôt une interprétation indéfinie (on) ou anaphorique.

\section{Méthodologie}

\subsection{Le corpus utilisé}

9 Le corpus choisi pour l'annotation est le French Treebank, ou FTB (Abeillé et al., 2003 et 2019). Il comprend des articles du journal Le Monde allant de 1990 à 1993, totalisant 
21550 phrases graphiques. Le corpus est richement annoté (catégories, fonctions, flexion, etc.) et comporte une version en dépendances (Candito et al., 2009 et 2010) annotée pour le passif, que nous utilisons dans cette étude. Dans le corpus, les passifs sont repérés grâce à l'annotation de l'auxiliaire être du passif par l'étiquette «aux.pass.».

\section{2. Élaboration du sous-corpus}

Nous avons extrait deux groupes de comparaison du FTB. Pour le premier groupe, nous avons extrait aléatoirement 125 verbes actifs transitifs à sujet nominal (désormais «actif SN») et 125 verbes passifs avec complément d'agent en par (désormais «passif long»). Pour le deuxième groupe, nous avons choisi d'extraire 125 actifs à sujet clitique (désormais «actif clitique») et 125 passifs sans agent exprimé (désormais "passif court»). Notre sous-corpus de travail comporte donc 500 phrases, 250 actives et 250 passives.

Nous avons choisi des phrases passives au passé composé ( SN a été SV [syntagme verbal]») de façon à privilégier les passifs verbaux face aux passifs adjectivaux («La porte est ouverte»). Nous avons choisi des compléments d'agent introduits par la préposition par, afin de ne pas ajouter un facteur de variation supplémentaire avec de. Les phrases à sujet infinitif ou phrastique sont peu nombreuses dans le corpus (Abeillé et al., 2019) et elles ne sont jamais au passif. Nous n'avons pas pris en compte les passifs impersonnels (exemple [6]), qui n'ont pas d'actifs impersonnels correspondants, et qui demanderaient une étude spécifique.

[6] Il a été décidé que désormais les programmes d'ajustements des flottilles seraient arrêtés par le Conseil des ministres.

(FTB, P. Lemaître, 22/12/1992)

De façon à obtenir des sous-corpus comparables, nous avons choisi des phrases actives au passé composé, avec un objet nominal ou pronominal. Le sujet des actifs clitiques est un pronom clitique, indéfini (on), anaphorique (il, elle...) ou déictique (nous, vous). Les pronoms ce, cela étaient également inclus ${ }^{1}$. Nous obtenons donc un parallèle entre l'argument 1 pronominal des actifs clitiques et l'argument 1 omis du passif court en ce que leur antécédent peut être présent dans le corpus.

Enfin, nous avons exclu à l'actif les phrases non passivables: verbe avoir (exemple [7]), locutions verbales (exemple [8]), objets numéraux (exemple [9]); ainsi que celles à sujet inversé, dans la mesure où elles ajouteraient une variable d'ordre des mots supplémentaire par rapport à l'alternance actif/passif.

[7] Pour l'instant, la rumeur n'a jamais eu le moindre début de confirmation. (FTB, 05/01/1993)

[8] Ce litige a donné lieu à une action [...].

(FTB, 09/02/1992)

1. Attal (1985) pointait la similitude entre passif court et actif dont le sujet est on. Toutefois, une telle restriction n'aurait pas permis de tester un facteur comme l'animéité, puisque on ne dénote que des humains. 
[9] Le taux de chômage a atteint 7,1 \% de la population active en décembre aux États-Unis.

(FTB, S. Marti, 13/01/1992)

Notre sous-corpus contient donc 500 phrases, avec un verbe au passé composé, sans inversion du sujet, avec passif court ou en par, à arguments nominaux ou pronominaux, et les actifs sont passivables. Pour assurer une comparaison robuste statistiquement, l'échantillonnage réalisé ne représente pas les proportions d'actifs et de passifs attestées dans le corpus. En effet, le passif reste une construction peu fréquente (5\% des verbes du corpus). Une autre possibilité aurait été de restreindre l'échantillon des phrases actives à un même ensemble de lemmes. Nous n'avons pas fait ce choix afin d'observer la répartition des verbes et des classes sémantiques selon la voix.

\subsection{Les facteurs annotés}

Nous utilisons 11 facteurs. Certains proviennent directement du corpus, d'autres ont été ajoutés pour les besoins de la présente étude. Le corpus est déjà annoté pour la personne, le genre, le nombre, la définitude des déterminants, la catégorie (SN, nom propre, pronom, clitique) et la fonction des arguments, le lemme verbal et le type de phrase (subordonnée, coordonnée). Nous annotons le caractère animé et la longueur des arguments, la classe sémantique du verbe d'après Dubois et DuboisCharlier (1997), l'amorçage (passif dans la phrase précédente) et avons précisé le type de phrase (principale, complétive, relative, type de circonstancielle).

Comme Bresnan et Ford (2010) dans leur étude sur l'alternance dative en anglais, et comme Thuilier (2012a) pour le français, nous calculons la longueur relative des arguments par la différence des valeurs logarithmiques de leur longueur en nombre de mots: $\log ($ Longueur $d u S N) \log ($ Longueur $d u S P)$. Nous utilisons les logarithmes de façon à réduire la dispersion (les arguments ont 1 à 30 mots) et à tenir compte de la distribution exponentielle du nombre de mots.

Concernant l'annotation de l'amorçage, contrairement à Estival (1985) et Weiner et Labov (1983) qui prennent les 5 phrases ou énoncés précédents en corpus oral, pour l'anglais, nous ne considérons que le passif dans la phrase qui précède, car nos phrases sont beaucoup plus longues. Contrairement à Estival (1985), nous ne distinguons pas amorçage lexical (le même verbe au passif) et amorçage syntaxique (un passif qui précède avec un autre verbe) (Bock, 1986). Dans la grande majorité des cas, l'amorçage était réalisé avec un verbe différent.

\section{Analyse statistique}

\subsection{Statistiques descriptives}

\subsubsection{L'argument omis du passif court}

Dans le groupe des passifs courts, nous avons pu inférer l'argument 1 omis dans $87 \%$ des cas (109 sur 125 phrases), ce qui a permis une annotation en genre, 
nombre, animéité et définitude. On trouve ainsi des arguments omis récupérés de façon anaphorique (exemples [10a] et [10b]). Dans ces cas, l'argument 1 du passif court est bien mis en correspondance avec un argument 1 clitique à l'actif («Ils ont embauché...», «Il a renvoyé...»).

[10a] [...] les responsables d'Euro Disney ont dû trouver une réponse rapide pour la formation du personnel hôtelier. Sept mille personnes ont, en effet, été embauchées pour assurer le service dans les 5200 chambres des six hôtels [...].

(FTB, F. Chirot, 08/07/1992)

[10b] Le FMI $_{i}$ aurait dû ouvrir une seconde ligne de crédit à la Russie en octobre, mais $\underline{i}_{i_{i}}$ différé sa décision devant les incertitudes croissantes sur la poursuite des réformes et la normalisation des relations au sein de la zone rouble. De même, la constitution d'un fonds de stabilisation du rouble de 6 milliards de dollars a été renvoyée à des temps meilleurs.

(FTB, M. A. Crosnier, 22/12/1992)

Notre observation en corpus confirme en partie les études descriptives sur le passif: l'argument 1 n'est pas exprimé en syntaxe mais il reste la plupart du temps implicite (Creissels, 2006; Jackendoff, 1977). Comme en [10], nous avançons par ailleurs que son interprétation est surtout anaphorique.

Contrairement à Attal (1985), les cas où l'argument omis s'interprète comme un humain indéfini, on ou quelqu'un, comme en [11], sont peu nombreux (8\% dans nos passifs courts).

[11a] Le Mouvement de défense des exploitants familiaux (MODEF), dont le président est Raymond Mineau, a été créé en avril 1959 à Toulouse.

(FTB, 07/07/1992)

[11b] M. Franck Riboud, 37 ans, a été nommé mardi 7 juillet directeur du développement de BSN.

(FTB, 09/07/1992)

Notons enfin que dans $5 \%$ des cas, l'argument 1 omis est totalement supprimé, ou en tout cas n'est pas récupérable en contexte (exemples [12a] et [12b]). Dans de tels cas, nous n'avons pas annoté d'argument 1 .

[12a] L'Afrique du Sud a enfin été confrontée à une forte hausse de ses coûts d'extraction. (FTB, 29/12/1992)

[12b] Percée également du bois [...], dont la part a été multipliée par deux, au détriment du charbon.

(FTB, F. Vaysse, 06/01/1992)

L'effacement de l'argument 1 n'est donc pas exclu, bien que plus rare que son maintien implicite. Hormis ces cas, nous avons donc noté tous les facteurs 
(nombre, genre, défini, humain, etc.) sur cet argument 1 implicite, sauf la longueur et la catégorie syntaxique.

\subsection{2. Écarts principaux entre actif et passif}

Le tableau 1 résume les écarts les plus importants entre actif et passif observés dans nos données. Ces résultats mettent en évidence plusieurs tendances.

\begin{tabular}{|l|r|r|r|r|r|}
\hline Facteurs & \multicolumn{2}{|c|}{ Actif } & \multicolumn{2}{|c|}{ Passif } & Total \\
\hline Arg1 animé & 219 & $53 \%$ & 192 & $47 \%$ & 411 \\
\hline Arg2 animé & 20 & $22 \%$ & 72 & $78 \%$ & 92 \\
\hline Arg1 défini & 230 & $56 \%$ & 181 & $44 \%$ & 411 \\
\hline Arg2 défini & 147 & $42 \%$ & 207 & $58 \%$ & 354 \\
\hline Arg2 pronominal & 38 & $42 \%$ & 53 & $58 \%$ & 91 \\
\hline Arg1 plus saillant que Arg2* & 68 & $75 \%$ & 23 & $25 \%$ & 91 \\
\hline Arg2 plus saillant que Arg1* & 7 & $13 \%$ & 46 & $87 \%$ & 53 \\
\hline Arg1 et Arg2 de même saillance* & 81 & $59 \%$ & 56 & $41 \%$ & 137 \\
\hline Longueur moyenne de Arg1* & 3 mots & 6 mots & \\
\hline Longueur moyenne de Arg2* & 5 mots & 3 mots & \\
\hline Arg1 plus long que Arg2* & 35 & $28 \%$ & 91 & $72 \%$ & 126 \\
\hline Arg2 plus long que Arg1* & 69 & $73 \%$ & 25 & $27 \%$ & 94 \\
\hline Arg1 et Arg2 de même longueur* & 21 & $70 \%$ & 9 & $30 \%$ & 30 \\
\hline Verbe de communication & 59 & $63 \%$ & 34 & $37 \%$ & 93 \\
\hline Verbe de contact & 11 & $31 \%$ & 24 & $69 \%$ & 35 \\
\hline Phrase précédée d'un passif & 70 & $36 \%$ & 162 & $64 \%$ & 196 \\
\hline * Ne prend en compte que les données $d u$ groupe «actif SN/passiflong». & & \\
\hline
\end{tabular}

Tableau 1 - Principaux écarts entre actif et passif au regard des facteurs annotés

Si l'on compare actif et passif, on constate que les arguments 1 animés et définis se trouvent majoritairement à l'actif (à $53 \%$ et $56 \%$ respectivement). À l'inverse, les arguments 2 animés, définis et pronominaux se trouvent surtout au passif (à $78 \%, 58 \%$ et $58 \%$ respectivement). Dans les deux cas, les arguments animés, définis et pronominaux occupent la fonction Sujet: l'argument 1 est sujet à l'actif et l'argument 2 l'est au passif. Cela confirme une tendance plus générale: les sujets sont préférentiellement définis, animés et pronominaux (Lambrecht, 1994). De là découlent les contrastes suivants, où « $\downarrow$ » indique une dégradation de l'acceptabilité due à une violation de contrainte préférentielle (notation empruntée à Miller, 2013) : 
[13a] Sujet animé: Un agriculteur a été blessé par une grenade lacrymogène. (FTB, 02/07/1992)

[13b] $\downarrow$ Sujet inanimé: Une grenade lacrymogène a blessé un agriculteur.

[14a] Sujet défini : Le principal responsable a été inculpé par un grand jury. (FTB, 03/07/1992)

[14b] $\downarrow$ Sujet indéfini: Un grand jury a inculpé le principal responsable.

[15a] Sujet pronominal: Ils avaient été condamnés par le tribunal de Saint-Étienne. (FTB, G. Buetas, 04/06/1993)

[15b] $\downarrow$ Sujet nominal: Le tribunal de Saint-Étienne les avait condamnés.

Nous proposons de rendre compte de la saillance informationnelle à partir de l'échelle suivante, proche de la proposition de Lambrecht (1994), qui prend en compte les marques de catégorie grammaticale (pronom, nom propre, syntagme nominal) et de définitude (défini/indéfini):

\section{Échelle de saillance: Pronom $<$ Nom propre $<S N$ défini $<S N$ indéfini}

Les marques formelles de pronominalité et de définitude correspondent en effet à une approximation, respectivement, de l'accessibilité (Faghiri et Thuilier, 2018) et de l'identifiabilité des référents (Lambrecht, 1994). Notre échelle reflète donc un codage des arguments qui va du plus identifiable et accessible (canoniquement, les pronoms) au moins identifiable et accessible (canoniquement, les SN indéfinis). Ce sont ces propriétés d'identifiabilité et d'accessibilité que nous appelons ici saillance². Cette échelle de saillance permet de tester si le sujet a tendance à coder l'argument le plus identifiable et accessible de la phrase. De fait, lorsque l'argument 1 est plus saillant que l'argument 2 , on observe à $75 \%$ une construction active, c'est-à-dire la construction qui permet à l'argument 1 d'être sujet. À l'inverse, si c'est l'argument 2 qui est le plus saillant, on observe une construction passive à $87 \%$, qui permet à nouveau à cet argument saillant d'occuper la fonction Sujet. Si les deux arguments partagent le même degré de saillance, on observe légèrement plus de phrases à l'actif (59\%), plaçant la construction active comme la construction par défaut vis-à-vis de ce facteur.

On observe également un effet de la longueur. Les arguments codés comme sujets (argument 1 à l'actif et argument 2 au passif) sont en moyenne plus courts que les arguments codés comme non sujets (argument 2 objet à l'actif et argument 1 oblique au passif). Les arguments longs sont plus fréquents lorsqu'ils ne sont pas sujets. Un argument 1 plus long que l'argument 2 donne ainsi à $72 \%$ une utilisation

2. Nous n'avons pas noté directement le statut informationnel en distinguant les référents déjà mentionnés et les référents nouveaux (opposition donné/nouveau), comme le font Weiner et Labov (1983) dans leur étude de corpus oral sur le passif en anglais. 
de la voix passive, qui permet à cet argument 1 long de ne pas être en position de sujet mais au contraire en position d'oblique postverbal. Si c'est l'argument 2 qui est le plus long, on a cette fois une construction active à $63 \%$, où cet argument 2 long est objet. Enfin, si la longueur des arguments est identique, on trouve $70 \%$ de phrases à l'actif, faisant de l'actif la construction par défaut pour ce facteur. Nous observons donc une contrainte «court avant long» dans nos données, qui semble influencer l'alternance actif/passif. D'où le contraste suivant:

[16a] Court avant long: Les magasins ont été créés par des gens qui étaient loin d'être tous des professionnels.

(FTB, P. Kremer, 05/01/1993)

[16b] $\downarrow$ Long avant court: Des gens qui étaient loin d'être tous des professionnels ont crée les magasins.

Les classes sémantiques permettent d'observer deux tendances: les verbes de communication sont plutôt utilisés à l'actif (à $63 \%$ ) tandis que les verbes de contact sont plutôt utilisés au passif (à $69 \%$ ). Ce dernier résultat fait écho à celui de Henley et al. (1995), qui montraient dans un corpus journalistique anglais, le Boston Globe, que les verbes de contact physique violent (par exemple en [17]) sont davantage construits au passif.

[17] Deux jeunes Français ont été assassinés, samedi 13 janvier, par des membres de l'organisation de guérilla Sentier lumineux.

(FTB, 01/1990)

Enfin, on remarque que si une phrase est précédée d'une forme passive, il y a plus de chance qu'elle soit au passif (à $64 \%$ ) qu'à l'actif. On interprète cet effet comme un effet d'amorçage syntaxique, ou persistance syntaxique: l'utilisation d'une structure syntaxique facilite sa réutilisation ultérieure (Bock, 1986), comme cela avait été observé par Estival (1985) sur un corpus oral anglais.

\subsubsection{L'effet de la saillance}

Nous avons défini la saillance informationnelle avec l'échelle suivante: Pronom $<$ Nom propre $<S N$ défini $<S N$ indéfini, qui permet d'approximer les notions d'accessibilité et d'identifiabilité à partir des marques formelles de catégorie grammaticale et de définitude.

Cette échelle permet une bonne modélisation de la proportion de passif dans nos données (figure 1), par une courbe de fonction exponentielle, dont le $\mathrm{R}^{2}$ est proche de $1\left(R^{2}=0,963\right)$.

On note ainsi une corrélation entre niveau de saillance et proportion de passif: plus l'argument 2 est saillant (pronom), plus le passif est utilisé. Inversement, moins il est saillant (SN indéfini), plus l'actif est utilisé. 


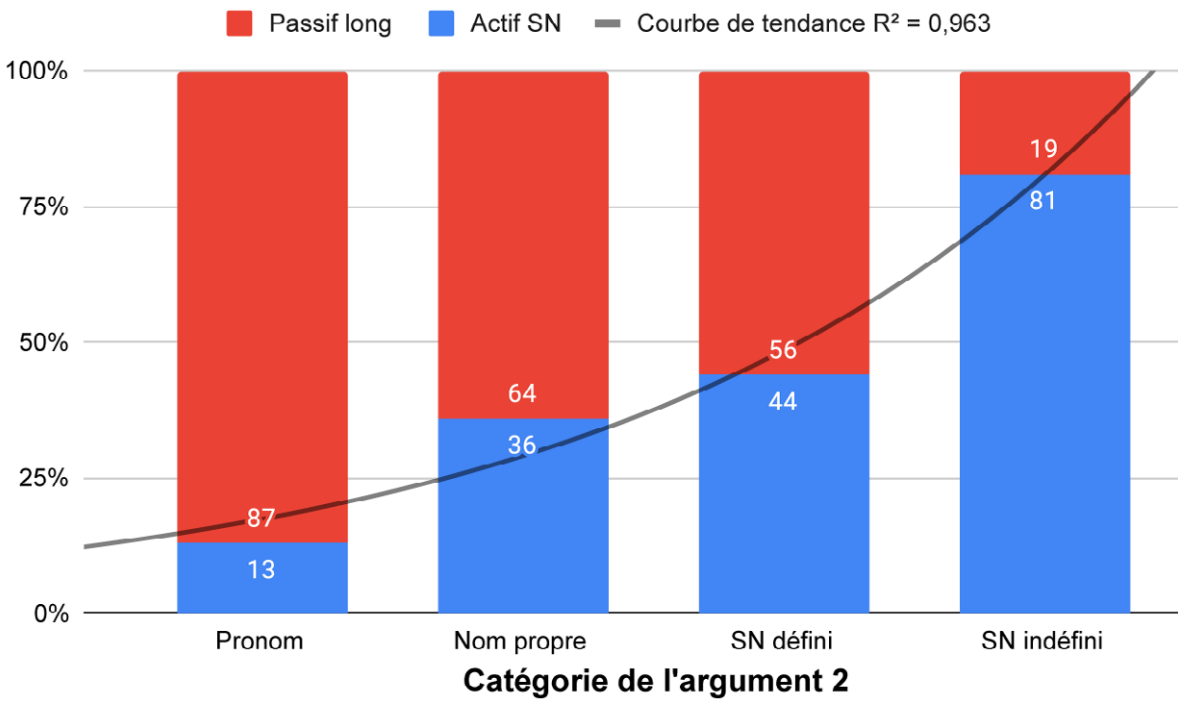

Figure 1 - Proportions d'actif/passif selon la saillance de l'argument 2, avec la courbe de la fonction exponentielle

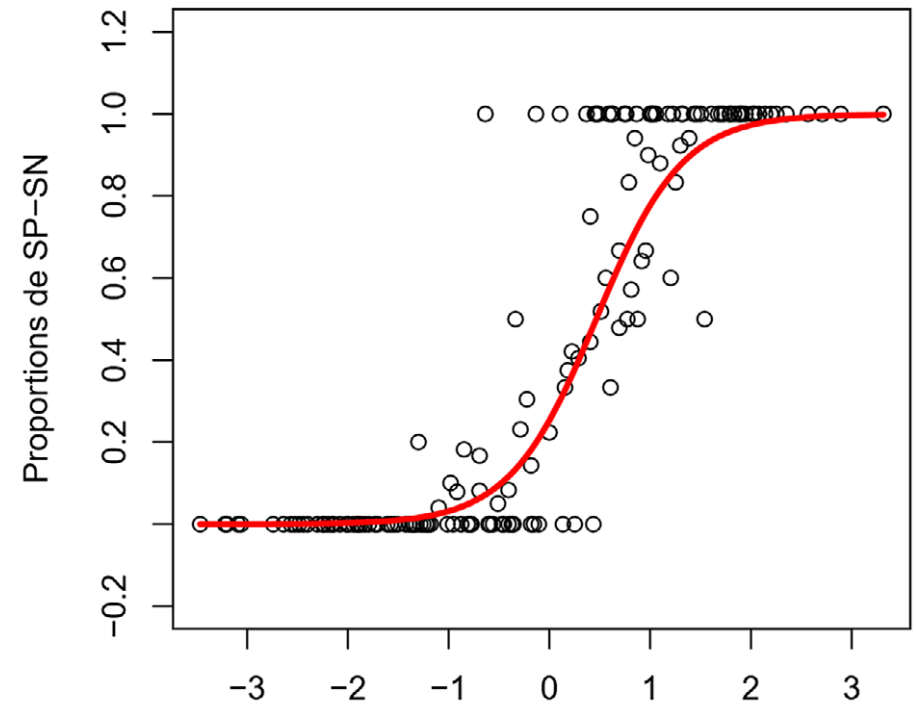

$\log ($ Longueur du SN) - $\log ($ Longueur du SP)

Figure 2 - Ordre des compléments postverbaux en fonction de leur longueur relative, avec la courbe de la fonction logistique (Thuilier, 2012a) 


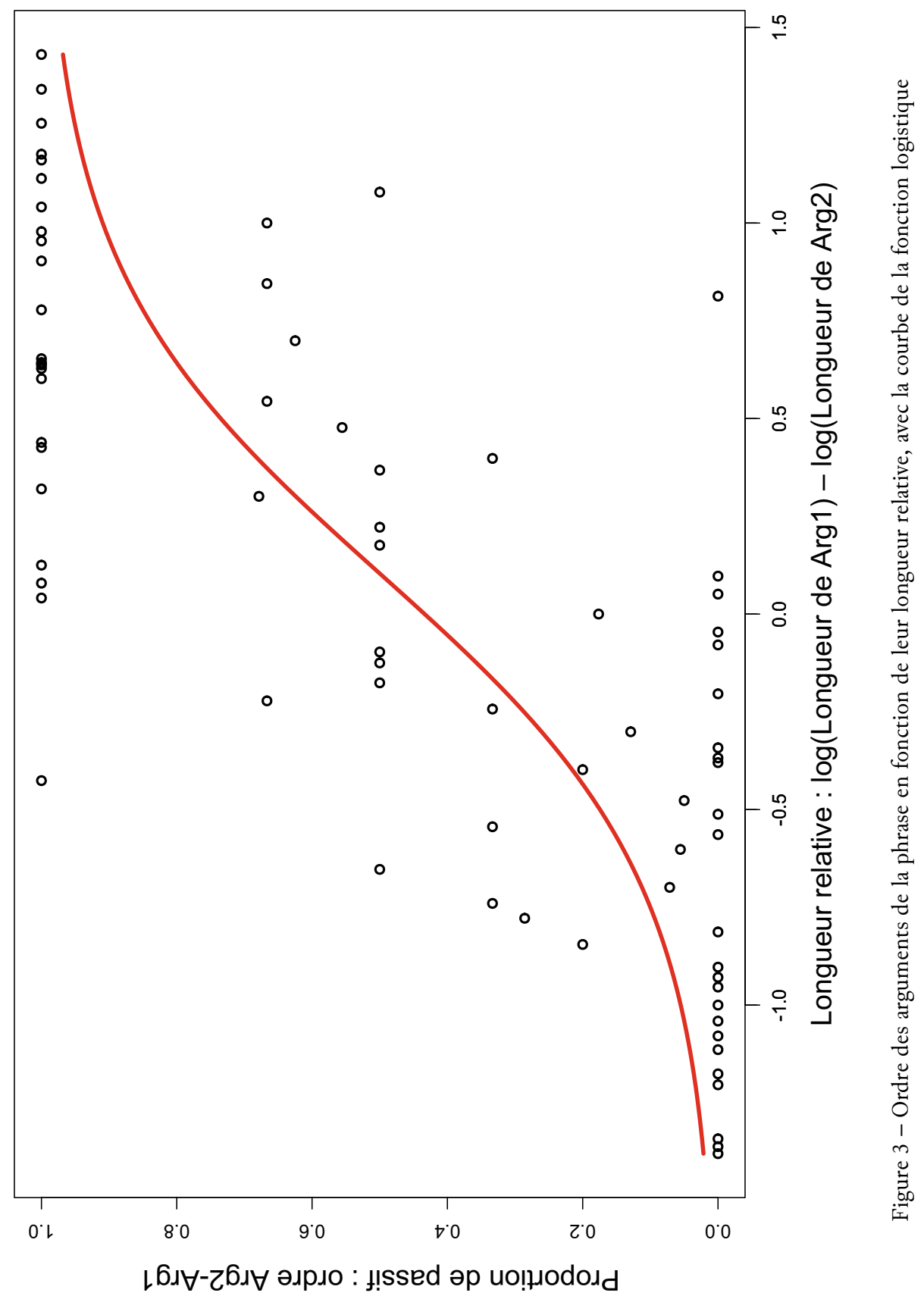




\subsubsection{L'effet de la longueur}

Thuilier (2012a) montre que la longueur relative (en nombre de mots) peut rendre compte de l'ordre relatif des compléments postverbaux au moyen de la fonction logistique. Cette fonction prend une valeur de 0 à 1 , est non linéaire, et sa représentation graphique est celle d'une courbe en $\mathrm{S}$ (figure 2).

Nous avons utilisé la même mesure de longueur relative (ici, log(Longueur de Arg1) $\log ($ Longueur de $\operatorname{Arg} 2))$ pour l'alternance d'ordre des arguments impliquée par la passivation (Arg1-V-Arg2 à l'actif, Arg2-V-par-Arg1 au passif). La modélisation des données requiert aussi la courbe de la fonction logistique. Nous mettons ainsi en évidence un parallèle entre le domaine postverbal et le domaine phrastique quant à l'effet de la longueur des constituants (figure 3 ).

\subsubsection{L'effet des subordonnées relatives}

En français comme dans d'autres langues (Keenan et Comrie, 1977), les relatives sujets sont plus fréquentes (Abeillé et al., 2001, pour le français; Roland et al., 2007, pour l'anglais) et généralement plus faciles à traiter que les relatives objets (Pozniak et Hemforth, 2015, pour le français; Gibson, 1998, pour l'anglais, par exemple). Nous attendons donc un effet de la relativation sur l'alternance actif/passif. La relativation de l'argument 2 à l'actif crée ainsi une relative objet, en que (exemple [18a]), mais au passif, cette même relativation de l'argument 2 crée une relative sujet, en qui (exemple [18b]). Sauf si le sujet est clitique ou inversé (Pozniak et al., à paraître), la relative en que est plus difficile à traiter du fait de l'intervention du sujet nominal entre l'argument 2 et le verbe.

[18a] Actif: Une question que les Français ont souvent soulevée [...].

[18b] Passif: Une question qui a souvent été soulevée par les Français [...].

(FTB, 1990)

La question se pose donc de savoir si le passif permet la relativation de l'argument 2 plus facilement, du fait que la relative sujet est préférée.

Le tableau 2 donne les résultats observés dans un échantillon indépendant de 125 verbes actifs à sujet non clitique, qui inclut des relatives sujets ${ }^{3}$.

\begin{tabular}{|l|r|r|r|r|}
\hline & \multicolumn{2}{|c|}{ Actif à sujet non clitique } & \multicolumn{2}{|c|}{ Passif long } \\
\hline Arg1 relatif & 13 & $10,4 \%$ & 0 & $0 \%$ \\
\hline Arg2 relatif & 2 & $1,6 \%$ & 17 & $13,6 \%$ \\
\hline
\end{tabular}

Tableau 2 - Nombre et proportion d'actif/passif selon l'argument relativé

3. Dans le groupe «actif $\mathrm{SN}$ » précédemment étudié, nous avions en effet exclu les relatives sujets, puisque leur contrepartie passive, en par qui par exemple, n'est pas attestée dans le corpus. 
À l'actif, les relatives objets (argument 2 relatif) apparaissent moins fréquentes que les relatives sujets (argument 1 relatif). Toutefois la relativation de l'argument 2 n'est pas rare pour autant, puisque le passif long permet justement de construire des relatives sujets avec l'argument 2 , préférées aux relatives objets. Le passif apparait donc ici comme une construction qui permet de satisfaire une contrainte plus générale sur les relatives.

Ce phénomène a été observé expérimentalement en anglais: la relative de l'argument 2 est plus facilement traitée lorsqu'elle est construite comme relative sujet au passif, plutôt que comme relative objet à l'actif (Gennari et MacDonald, 2008).

\subsection{Statistiques inférentielles}

La modélisation statistique a été effectuée sur le logiciel $\mathrm{R}$ via la fonction glmer binomiale (régression logistique à effets mixtes) du package «lme4». La variable à prédire est la variable binomiale Vorx. Elle vaut 1 en cas de passif et 0 en cas d'actif. Les variables prédictives ont été normalisées avec la fonction scale. Un coefficient positif favorise le passif (c'est-à-dire augmente sa probabilité d'après le modèle) et un coefficient négatif favorise l'actif. La variable LEMME est utilisée comme variable aléatoire ${ }^{4}$, de manière à tenir compte de l'effet lexical individuel du verbe sur la préférence entre actif et passif. Les interactions entre facteurs ont été testées et ne sont pas significatives ${ }^{5}$.

Nous avons établi trois modèles à partir de nos données: un modèle Passiflong pour le groupe "actif SN/passif long» (tableau 3), un modèle Passif court pour le groupe «actif clitique/passif court» (tableau 4), et un modèle Actif/Passif pour l'ensemble des données (tableau 5).

L'évaluation des modèles a été réalisée par la mesure de leur exactitude (ratio entre les prédictions correctes et le nombre total de prédictions). Utilisés en prédiction sur nos données, les modèles retournent pour chaque phrase une valeur $p$ de probabilité de passif. Le seuil de prédiction est fixé à 0,5 : le modèle prédit un passif si $p>0,5$ et il prédit un actif sinon.

Ces modèles permettent de mettre en évidence le caractère significatif de certaines tendances dégagées lors de l'analyse descriptive. Notamment:

- dans tous les modèles, un argument 2 animé et défini favorise significativement le passif, c'est-à-dire son codage comme sujet;

- dans les modèles Passif long et Actif/Passif, le passif est favorisé lorsque l'argument 2 est plus saillant que l'argument 1 ;

- l'effet de la longueur est significatif dans les modèles Passiflong et Actif/Passif, où un argument 1 plus long que l'argument 2 favorise le passif, c'est-à-dire son codage comme oblique postverbal;

- dans le modèle Passif court, l'amorçage passif, c'est-à-dire le fait d'être précédé par une forme passive, favorise le passif.

4. L'auteur des articles n'a pas été utilisé comme variable aléatoire car certains articles du Monde sont collectifs ou simplement signés «Le Monde».

5. Dans la dernière colonne des tableaux ci-après, le seuil de $p$-value indique que les facteurs sont significatifs. 


\begin{tabular}{|l|r|r|}
\hline & Coefficient estimé & $p$-value \\
\hline Intercept & $-2,36$ & $<0,001$ \\
\hline Arg1 plus long que Arg2 & 1,90 & $<0,001$ \\
\hline Arg2 défini & 1,84 & $<0,001$ \\
\hline Arg2 plus saillant que Arg1 & 1,66 & $<0,001$ \\
\hline Arg2 animé & 1,42 & $<0,01$ \\
\hline Arg2 pluriel & 1,2 & $<0,01$ \\
\hline Arg1 pluriel & 1,2 & $<0,01$ \\
\hline Exactitude & $82 \%$ & \\
\hline
\end{tabular}

Tableau 3 - Résultats du modèle Passif long, obtenu par régression logistique sur le groupe «actif SN/passif long»

\begin{tabular}{|l|r|r|}
\hline & Coefficient estimé & $p$-value \\
\hline Intercept & $-1,47$ & $<0,001$ \\
\hline Arg1 féminin & 1,33 & $<0,001$ \\
\hline Arg2 animé & 1,32 & $<0,01$ \\
\hline Arg2 relatif & $-1,18$ & $<0,01$ \\
\hline Arg2 défini & 0,87 & $<0,05$ \\
\hline Passif amorcé & 0,86 & $<0,01$ \\
\hline Arg2 pluriel & 0,71 & $<0,05$ \\
\hline Exactitude & \multicolumn{2}{|c|}{$69 \%$} \\
\hline
\end{tabular}

Tableau 4 - Résultats du modèle Passif court, obtenu par régression logistique sur le groupe «actif clitique/passif court»

\begin{tabular}{|l|r|r|}
\hline & Coefficient estimé & $p$-value \\
\hline Intercept & $-6,39$ & $<0,5$ \\
\hline Arg1 plus long que Arg2 & 1,91 & $<0,001$ \\
\hline Arg2 défini & 1,79 & $<0,001$ \\
\hline Arg2 plus saillant que Arg1 & 1,67 & $<0,001$ \\
\hline Arg2 animé & 1,42 & $<0,01$ \\
\hline Arg2 pluriel & 1,21 & $<0,01$ \\
\hline Arg1 pluriel & 1,19 & $<0,01$ \\
\hline Exactitude & $88 \%$ & \\
\hline
\end{tabular}

Tableau 5 - Résultats du modèle Actif/Passif, obtenu par régression logistique sur la totalité des données 
Certains facteurs ont en revanche un effet que les seules statistiques descriptives ne mettaient pas en évidence.

De façon un peu inattendue, le modèle Passif court met en évidence un effet significatif du genre grammatical: un argument 1 féminin favorise le passif. Cet effet peut être imputé à la surreprésentation des référents masculins comme arguments 1 sujets dans le groupe «actif clitique» ( $83 \%$ des arguments 1 du groupe sont masculins). Nous faisons l'hypothèse que cela est dû à une interaction du genre grammatical, du genre social, et du genre textuel: les pronoms masculins dénotent le plus souvent des hommes dans ce corpus (dans le groupe «actif clitique», $92 \%$ des pronoms sujets masculins désignent des hommes), et le discours journalistique se fait davantage au sujet d'hommes que de femmes, comme l'ont montré Huet et al. (2013) sur le journal Le Monde dans les années précédant de peu celles des extraits du FTB ${ }^{6}$.

Le nombre a également un effet inattendu dans nos données: un argument au pluriel favorise le passif dans l'ensemble des modèles. L'interaction entre «Arg1 pluriel» et «Arg2 pluriel» n'est pas significative. Nous n'avons pas d'explication quant à ce fait.

Enfin, toujours dans le modèle Passif court, le fait que l'argument 2 soit relatif favorise l'actif. Cela semble aller contre les observations que nous avions faites sur les relatives (tableau 2), où nous avancions que les relatives sujets étaient préférées, et que la relativation de l'argument 2 favorisait donc le passif. Le résultat que l'on obtient ici est dû au sujet clitique, qui améliore le traitement de la relative objet. En effet, une partie des difficultés de traitement de la relative objet lorsque le sujet est un SN tient à l'effet d'intervention du sujet nominal entre l'antécédent de la relative et le verbe (Pozniak et al., à paraitre; Gibson, 1998 et 2000).

Toutefois, lorsque le sujet est un pronom comme en [19], l'effet d'intervention disparait et le nombre de relatives objets observées en corpus est alors plus important. On trouve ainsi $18 \%$ de relatives objets dans le groupe «actif clitique», mais seulement $1,6 \%$ dans le groupe «actif SN».

[19] Il sait de quoi il parle: il se bat pour faire vivre une boulangerie et un restaurant qu'il a créés.

(FTB, M.-C. Betbeder, 02/06/1993)

\section{Discussion}

Outre le rôle des contraintes cognitives plus générales, comme l'amorçage syntaxique, qui a été observé pour d'autres langues (Bock, 1986; Estival, 1985), notre étude montre la prépondérance de deux facteurs dans l'alternance actif/passif.

6. Ce privilège du masculin est peut-être plus général. Dans le grand corpus oral ANCOR («Anaphore et coréférence dans les corpus oraux») annoté pour les relations anaphoriques, Lefeuvre et al. (2014) ont trouvé une surreprésentation des reprises (nominales et pronominales) au masculin (53\% contre $43 \%$ au féminin et $4 \%$ de genre inconnu). 
- La structure informationnelle: la fonction Sujet code les référents les plus saillants (c'est-à-dire, accessibles et identifiables). En ce sens, la passivation apparait comme un processus de topicalisation de l'argument 2 , du fait que le sujet constitue le topique canonique (Lambrecht, 1994). Le passif assure ainsi la continuité du topique (Givón, 1983).

- La longueur: les constituants courts apparaissent préférentiellement avant les constituants longs. Le passif permet ainsi de contourner la pénalité sur les sujets longs en codant un argument 1 long comme un oblique postverbal. La longueur du sujet est en effet coûteuse cognitivement, en termes de mémoire de travail et de prédiction syntaxique (Chen et al., 2005 ; Gibson, 1998 et 2000). Cette contrainte se retrouve dans le domaine postverbal en français, où Thuilier (2012a) aboutit à des modélisations similaires au moyen de la fonction logistique.

L'effet de la structure informationnelle pointe des similarités entre langues sur la construction passive. Sur leur corpus oral en anglais, Weiner et Labov (1983) montrent ainsi que le caractère donné de l'argument 2 augmente significativement la proportion de passifs.

Plus généralement, à l'image de ce que Bresnan et Ford (2010) proposaient pour l'alternance dative en anglais, nous montrons le rôle de l'alignement harmonique des arguments pour l'alternance actif/passif en français.

La figure 4 établit ainsi les contraintes préférentielles mises au jour dans nos données. Le symbole «<» indique une relation de précédence linéaire entre les porteurs des propriétés, par exemple un argument défini tend à précéder un argument indéfini. Les arguments s'alignent alors selon ces contraintes pour minimiser les violations, d'où l'expression d'alignement harmonique.

Les contraintes sont présentées dans l'ordre croissant de leur coefficient d'après le modèle Actif/Passif (tableau 5). La pronominalité n'est pas significative dans ce modèle en partie parce que son effet est corrélé à celui de la saillance (coefficient $=0,74$ ). L'échelle de saillance proposée inclut en effet déjà la distinction pronominal/non pronominal. Toutefois, dans une modélisation où la pronominalité remplace la saillance, on lui observe également un effet significatif («Arg2 pronominal» est associé à $z=2,2 ; p<0,05)$.

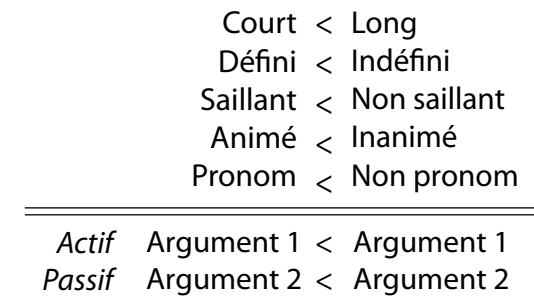

Figure 4 - Contraintes préférentielles de l'alternance actif/passif sous forme d'alignement harmonique 
L'intérêt de la modélisation par l'alignement harmonique est que toutes les contraintes s'appliquent simultanément. Ainsi, leurs effets peuvent se renforcer. Par exemple, un argument 1 court, pronominal et animé avec un argument 2 long, nominal et inanimé facilite le choix pour une construction active (exemple [20]). Les effets peuvent également être contradictoires. C'est le cas si un argument animé est long ou qu'un inanimé est court (exemple [21]). Dans ce cas, les coefficients associés au modèle sont importants pour déterminer le poids accordé à chaque contrainte dans le choix entre actif et passif. Dans notre modélisation, le poids de la contrainte de longueur est ainsi plus fort que le poids du caractère animé.

[20] Et puis ils avaient initialement négligé les phénomènes monétaires et notamment la baisse du dollar, qui n'a pas joué en faveur de la compétitivité des entreprises françaises.

(FTB, 05/01/1993)

[21] D'entrée, les rôles avaient été répartis par M. Perigot qui, par exemple, accorda «une minute et demie» à M. Guillen pour qu'il expose l'engagement de l'UIMM [...].

(FTB, A. Lebaude et P. Guillen, 09/06/1993)

Ces types de contraintes, formalisables de manière plus explicite dans le cadre de la «Théorie de l'optimalité stochastique» (Bresnan et al., 2001), ont pu être observés dans d'autres langues (Kempen et Harbusch, 2004; Branigan et Feleki, 1999; Tanaka et al., 2011), ce qui tend vers l'idée de contraintes cognitives générales qui pèsent sur les grammaires des langues du monde. Notons par ailleurs que ces contraintes sont hétérogènes et ne sont pas simplement d'ordre syntaxique. Ainsi l'animéité est un facteur sémantique, tandis que la définitude est liée au statut informationnel.

De plus, les différences entre langues et/ou entre constructions peuvent être représentées par la différence de poids que chaque langue ou chaque construction assigne aux contraintes. Ainsi, dans leur modèle de l'alternance dative en anglais, Bresnan et Ford (2010) montrent que la contrainte d'animéité est plus forte que celle de définitude. Dans notre modélisation de l'alternance actif/passif en français, nous observons l'inverse. De même, Thuilier (2012a) ne note pas d'effet de l'animéité dans l'ordre des compléments postverbaux en français, tandis que nous observons un effet de ce facteur dans notre modélisation.

Par ailleurs, notre modèle montre que l'argument 1 du passif long, le complément d'agent, est soumis en tant qu'argument à part entière aux contraintes d'alignement harmonique. Notre étude pointe donc vers une analyse du passif qui intègre le complément d'agent à la structure argumentale du verbe, au même titre que le sujet. Dans la théorie minimaliste (Chomsky, 1995; Pollock, 1997; Rouveret, 2015), la phrase passive est dérivée par deux opérations: d'une part la suppression de l'argument 1, qui permet le mouvement de l'argument 2 en position de sujet, d'autre part l'adjonction du complément d'agent en tant que modifieur optionnel, dont le 
rôle sémantique est assigné par la préposition par. Certaines versions de grammaire catégorielle suivent également cette analyse (Desclés et Guentcheva, 1993).

Nous mettons ainsi en cause cette analyse, qui ne permet pas de saisir le complément d'agent en tant qu'argument légitimé par le verbe passif, et qui en reçoit son rôle sémantique. Les grammaires d'unification (LFG [Lexical Functional Grammar - grammaire lexicale fonctionnelle], HPSG [Head-Driven Phrase Structure Grammar - grammaire syntagmatique guidée par les têtes], voir Abeillé, 2007) formalisent au contraire la passivation comme une alternance de valence, où le réappariement entre fonctions syntaxiques et rôles sémantiques au passif permet au complément d'agent d'être sélectionné par le verbe passif. Ainsi, la règle lexicale utilisée en HPSG (figure 5) intègre le complément d'agent «SP $\mathrm{S}_{\mathrm{i}}$ » la structure argumentale «ST-ARG» du verbe passif (Bresnan, 1982; Flickinger, 1987; Müller, 2000).

Même si leur approche est moins lexicale, les grammaires de construction aussi intègrent le complément d'agent à la structure argumentale du passif (Goldberg, 1995).

Enfin, cette étude en appelle d'autres. Une étude comparable pourrait être conduite avec des compléments d'agent en $d e$. Le rôle du lemme verbal pourrait être étudié en extrayant des phrases actives et passives avec les mêmes lemmes. L'effet de genre - comme de nombre - est nouveau, et n'a pas été rapporté pour le passif d'autres langues, à notre connaissance, et serait à explorer davantage. Nos résultats seraient également à corroborer par des études sur d'autres corpus et par des études expérimentales ${ }^{7}$.

Le choix du corpus journalistique (FTB) était motivé par le fait qu'il était annoté pour le passif, mais il ne nous a pas permis d'observer un effet de la personne, contrairement à Bresnan et al. (2001) sur l'anglais, car la troisième personne y est massivement utilisée. De ce fait, recourir à des corpus dialogiques (oraux ou écrits informels) permettrait d'étudier le facteur de la personne.

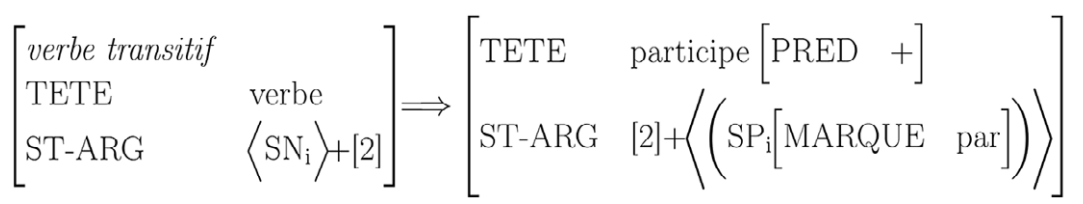

Figure 5 - Règle lexicale du passif en HPSG

7. Thuilier et al. (à paraître) ont mis en évidence le rôle du caractère animé des arguments dans une expérience de rappels de phrases en français: les phrases passives sont plus souvent répétées à l'actif si l'argument 2 est inanimé ( $25 \%$ de changement de voix) que s'il est animé (7,4\% de changement de voix). Cela montre que le passif est défavorisé lorsque le sujet est inanimé, ce que nous montrons ici également. 


\section{Conclusion}

Notre étude a permis de mettre en évidence de nouvelles caractéristiques de l'alternance actif/passif en français écrit, et cela grâce à une approche basée sur la modélisation statistique des données de corpus.

D'un point de vue descriptif, nous observons que $5 \%$ des verbes du FTB sont au passif (contre $9 \%$ en anglais pour le même type de texte, voir Roland et al., 2007) et que le passif long représente environ un quart des usages du passif à l'écrit (contre $10 \%$ à l'oral, voir Hamma et al., 2017). Nous avons également mis en évidence certains facteurs significatifs qui déterminent le choix de la voix: animéité, définitude, pronominalité, saillance et longueur des arguments. Certains de ces facteurs ont pu être observés dans d'autres langues et pour d'autres constructions : l'animéité dans le passif en japonais (Tanaka et al., 2011), la définitude, la pronominalité, l'animéité et la longueur dans l'alternance dative en anglais (Bresnan et Ford, 2010), la longueur dans la complémentation postverbale en français (Thuilier, 2012a). Notre étude va donc dans le sens de la théorie de l'alignement harmonique, qui prévoit que les mêmes contraintes d'ordre préférentiel soient à l'œuvre dans toutes les langues, indépendamment des fonctions syntaxiques. L'espace de variation entre les langues se situe alors dans le poids accordé à chaque contrainte, qui peut également varier selon les constructions. Ainsi en français, alors que la complémentation postverbale ne semble pas accorder de poids à l'animéité (Thuilier, 2012a), nous montrons que l'alternance actif/passif y est soumise. Le domaine de la phrase et le domaine postverbal ne seraient ainsi pas soumis aux mêmes contraintes en français.

Du point de vue théorique, notre étude sur corpus montre que l'argument 1 du passif court n'est pas forcément supprimé mais reste le plus souvent seulement implicite (anaphorique et récupérable en contexte dans près de $80 \%$ des passifs courts). L'agent reste donc impliqué par le verbe passif, ce qui distingue le passif des constructions moyennes ou médiopassives (Creissels, 2006; Jackendoff, 1977). Ce caractère implicite de l'argument 1 omis au passif court rejoint l'analyse du complément d'agent du passif long comme un argument à part entière. Nous avons en effet montré que le complément d'agent est soumis aux contraintes préférentielles au même titre que le sujet. Cela soutient ainsi les théories syntaxiques qui intègrent ce complément à la structure argumentale du verbe (comme LFG ou HPSG, voir Bresnan, 1982; Flickinger, 1987; Müller, 2000).

\section{Références bibliographiques}

Abeillé, A. 2007. Les grammaires d'unification. Paris: Hermes Science-Lavoisier.

Abeillé, A., Clément, L., Kinyon, A. et Toussenel, F. 2001. Un corpus français arboré: quelques interrogations. In Actes de la $8^{e}$ conférence annuelle sur le Traitement automatique des langues naturelles (TALN - RECITAL 2001). Paris: Association pour le Traitement automatique des langues (ATALA) : 33-42. En ligne à l'adresse suivante: https://www. atala.org/sites/default/files/actes_taln/AC_0025.pdf. 
Abeillé, A., Clément, L. et LiÉgeois, L. 2019. Un corpus arboré pour le français: le French Treebank. TAL - Traitement automatique des langues 60 (2): 19-43. En ligne à l'adresse suivante: https://www.atala.org/sites/default/files/TAL-60-2-1Abeille.pdf.

Abeillé, A., Clément, L. et Toussenel, F. 2003. Building a Treebank for French. In A. Abeillé (éd.), Treebanks: Building and Using Parsed Corpora. Dordrecht - Boston Londres: Kluwer Academic Publishers: 165-187.

Attal, P. 1985. Le passif: étude de corpus. L'information grammaticale 27: 10-13.

BaAyen, R. H. 2008. Analyzing Linguistic Data: A Practical Introduction to Statistics Using R. Cambridge - New York - Melbourne: Cambridge University Press.

Bock, J. K. 1986. Syntactic Persistence in Language Production. Cognitive Psychology 18 (3): 355-387.

Branigan, H. P. et Feleki, E. 1999. Conceptual Accessibility and Serial Order in Greek Speech Production. In M. Hahn et S. C. Stoness (éd.), Proceedings of the 21st Annual Conference of the Cognitive Science Society. New York: Psychology Press: 96-101.

Bresnan, J. 1982. The Passive in Lexical Theory. In J. Bresnan (éd.), The Mental Representation of Grammatical Relations. Cambridge - Londres : MIT Press: 3-86.

Bresnan, J., Cueni, A., Nikitina, T. et Bahyen, R. H. 2007. Predicting the Dative Alternation. In G. Bouma, I. Krämer et J. Zwarts (éd.), Cognitive Foundations of Interpretation. Amsterdam: Royal Netherlands Academy of Arts and Sciences: 69-94.

Bresnan, J., Dingare, S. et Manning, C. D. 2001. Soft Constraints Mirror Hard Constraints: Voice and Person in English and Lummi. In M. Butt et T. Holloway King (éd.), Proceedings of the LFG'01 Conference. Stanford: CSLI Publications: 13-32. En ligne à l'adresse suivante: http://web.stanford.edu/group/cslipublications/cslipublications/ LFG/6/pdfs/lfg01bresnanetal.pdf.

Bresnan, J. et Ford, M. 2010. Predicting Syntax: Processing Dative Constructions in American and Australian Varieties of English. Language 86 (1): 168-213.

Bresnan, J. et Nikitina, T. 2009. The Gradience of the Dative Alternation. In L. Uyechi et L.-H. WeE (éd.), Reality Exploration and Discovery: Pattern Interaction in Language and Life. Stanford: CSLI Publications: 161-184.

Candito, M., Crabbé, B. et Denis, P. 2010. Statistical French Dependency Parsing: Treebank Conversion and First Results. In N. Calzolari, K. Choukri, B. Maegaard, J. Mariani, J. Odijk, S. Piperidis, M. Rosner et D. Tapias (éd.), Proceedings of the 7th International Conference on Language Resources and Evaluation - LREC 2010. Paris: European Language Resources Association (ELRA) : 1840-1847. En ligne à l'adresse suivante: http://www.lrec-conf.org/proceedings/lrec2010/pdf/392_Paper.pdf.

Candito, M., Crabbé, B., Denis, P. et Guérin, F. 2009. Analyse syntaxique du français: des constituants aux dépendances. In $16^{e}$ conférence sur le Traitement automatique des langues naturelles (TALN 2009, Senlis, 24-26 juin 2009). En ligne sur HAL: https:// hal.archives-ouvertes.fr/hal-00495287.

Chen, E., Gibson, E. et Wolf, F. 2005. Online Syntactic Storage Costs in Sentence Comprehension. Journal of Memory and Language 52 (1): 144-169.

Chomsкy, N. 1995. The Minimalist Program. Cambridge - Londres: MIT Press. 
Creissels, D. 2006. Syntaxe générale: une introduction typologique. 2, La phrase. Paris: Hermes Science-Lavoisier.

Desclés, J.-P. et Guentchéva, Z. 1993. Le passif dans le système des voix du français. Langages 109: 73-102.

Dubois, J. et Dubois-Charlier, F. 1997. Les verbes français. Paris: Larousse. Version informatisée en ligne à l'adresse suivante : http://rali.iro.umontreal.ca/rali/?q=fr/versionsinformatisees-lvf-dem.

Estival, D. 1985. Syntactic Priming of the Passive in English. Text 5 (1-2): 7-21.

FAGHiri, P. et Thuilier, J. 2018. Ordre des compléments postverbaux en français : poids et accessibilité discursive. In F. Neveu, B. Harmegnies, L. Hriba et S. Prévost (éd.), SHS Web of Conferences. Actes du $6^{e}$ congrès mondial de Linguistique française-CMLF 2018 (Université de Mons, Belgique, 9-13 juillet 2018). Les Ulis: EDP Sciences. Vol. 46 : 1-15. En ligne à l'adresse suivante : https://www.shs-conferences.org/articles/shsconf/ pdf/2018/07/shsconf_cmlf2018_14008.pdf.

Flickinger, D. P. 1987. Lexical Rules in the Hierarchical Lexicon. Thèse de doctorat. Stanford University.

GAATONE, D. 1993. Les locutions verbales et les deux passifs du français. Langages 109: 37-52.

GaAtone, D. 1998. Le passif en français. Bruxelles: Duculot.

Gennari, S. P. et MacDonald, M. C. 2008. Semantic Indeterminacy in Object Relative Clauses. Journal of Memory and Language 58 (2): 161-187.

Gibson, E. 1998. Linguistic Complexity: Locality of Syntactic Dependencies. Cognition $68(1): 1-76$.

Gibson, E. 2000. The Dependency Locality Theory: A Distance-Based Theory of Linguistic Complexity. In A. Marantz, Y. Miyashita et W. O’Neil (éd.), Image, Language, Brain: Papers from the First Mind Articulation Project Symposium. Cambridge - Londres: MIT Press: 94-126.

Givón, T. (éd.) 1983. Topic Continuity in Discourse: A Quantitative Cross-Language Study. Typological Studies in Language 3. Amsterdam - Philadelphie: J. Benjamins.

Goldberg, A. E. 1995. Constructions: A Construction Grammar Approach to Argument Structure. Chicago - Londres: University of Chicago Press.

Hamma, B., Tardif, A. et Badin, F. 2017. Le passif à l'oral. In P. Larrivée et F. Lefeuvre (éd.), Français contemporain vernaculaire (FRACOV). 1-15. En ligne à l'adresse suivante: http://www.univ-paris3.fr/medias/fichier/passif-a-1-oral_1486478858794.pdf.

Henley, N. M., Miller, M. et Beazley, J. A. 1995. Syntax, Semantics, and Sexual Violence: Agency and the Passive Voice. Journal of Language and Social Psychology 14 (1-2): 60-84.

Huet, T., Biega, J. et Suchanek, F. M. 2013. Mining History with "Le Monde". In F. M. Suchanek et S. Riedel (éd.), Proceedings of the 2013 Workshop on Automated Knowledge Base Construction - AKBC'13. New York: Association for Computing Machinery: 49-54.

Jackendoff, R. S. 1977. X Syntax: A Study of Phrase Structure. Linguistic Inquiry Monographs 2. Cambridge - Londres: MIT Press. 
Keenan, E. L. et Comrie, B. 1977. Noun Phrase Accessibility and Universal Grammar. Linguistic Inquiry 8 (1) : 63-99.

Kempen, G. et Harbusch, K. 2004. A Corpus Study into Word Order Variation in German Subordinate Clauses: Animacy Affects Linearization Independently of Grammatical Function Assignment. In T. Pechmann et C. Habel (éd.), Multidisciplinary Approaches to Language Production. Berlin - New York: Mouton de Gruyter: 173-181.

Lambrecht, K. 1994. Information Structure and Sentence Form: Topic, Focus, and the Mental Representations of Discourse Referents. Cambridge Studies in Linguistics 71. Cambridge - New York - Melbourne: Cambridge University Press.

LeClère, C. 1993. Classes de constructions directes sans passif. Langages 109: 7-31.

Lefeuvre, A., Antoine, J.-Y. et Schang, E. 2014. Le corpus ANCOR_Centre et son outil de requêtage: application à l'étude de l'accord en genre et nombre dans les coréférences et anaphores en français parlé. In F. Neveu, P. Blumenthal, L. Hriba, A. Gerstenberg, J. Meinschaefer et S. Prévost (éd.), SHS Web of Conferences. Actes $d u 4^{e}$ congrès mondial de Linguistique française - CMLF 2014 (Berlin, 19-23 juillet 2014). Les Ulis: EDP Sciences. Vol. 8 : 2691-2706. En ligne à l'adresse suivante: https://www. shs-conferences.org/articles/shsconf/pdf/2014/05/shsconf_cmlf14_01359.pdf.

Mathieu, Y. 1993. Quelques passifs avec agent obligatoire. Langages 109: 35-36.

Miller, P. 2013. Usage Preferences: The Case of the English Verbal Anaphor “do so”. In S. Müller (éd.), Proceedings of the 20th International Conference on Head-Driven Phrase Structure Grammar - HPSG 2013. Stanford: CSLI Publications: 121-139. En ligne à l'adresse suivante: http://web.stanford.edu/group/cslipublications/cslipublications/ HPSG/2013/miller.pdf.

Müller, S. 2000. The Passive as a Lexical Rule. In D. Flickinger et A. Kathol (éd.), Proceedings of the 7th International Conference on Head-Driven Phrase Structure GrammarHPSG 2000. Stanford: CSLI Publications: 247-266. En ligne à l'adresse suivante: http:// web.stanford.edu/group/cslipublications/cslipublications/HPSG/2000/hpsg00mueller.pdf.

Pollock, J.-Y. 1997. Langage et cognition. Introduction au programme minimaliste de la grammaire générative. Paris: PUF.

Pozniak, C. et Hemforth, B. 2015. Processing Subject and Object Relative Clauses in French and Mandarin Chinese. In 28tb CUNY Conference on Human Sentence Processing (University of Southern California, Los Angeles, March 19-21, 2015). Poster en ligne à l'adresse suivante: https://www.researchgate.net/profile/Barbara_Hemforth/ publication/273765462_Processing_subject_and_object_relative_clauses_in_French_ and_Mandarin_Chinese/links/550b66700cf290bdc111e73c/Processing-subject-andobject-relative-clauses-in-French-and-Mandarin-Chinese.pdf.

Pozniak, C., Hemforth, B. et Abeillé, A. à paraître. Subject Inversion in French Object Relatives: What's Your Preference? In B. Crysmann et M. SaIler (éd.), One-to-Many Relations in Morphology, Syntax, and Semantics. Berlin: Language Science Press : 153-177.

Roland, D., Dick, F. et Elman, J. L. 2007. Frequency of Basic English Grammatical Structures: A Corpus Analysis. Journal of Memory and Language 57 (3) : 348-379.

Rouveret, A. 2015. Arguments minimalistes: une présentation du Programme Minimaliste de Noam Chomsky. Lyon: ENS Éditions. 
Tanaka, M. N., Branigan, H. P., McLean, J. F. et Pickering, M. J. 2011. Conceptual Influences on Word Order and Voice in Sentence Production: Evidence from Japanese. Journal of Memory and Language 65 (3): 318-330.

Thuilier, J. 2012a. Contraintes préférentielles et ordre des mots en français. Thèse de doctorat. Université Paris Diderot - Paris 7.

Thuilier, J. 2012b. Lemme verbal et classe sémantique dans l'ordonnancement des compléments postverbaux. In F. Neveu, V. Muni Toke, P. Blumenthal, T. Klingler, P. Ligas, S. PrÉvost et S. Teston-Bonnard (éd.), SHS Web of Conferences. Actes du $3^{e}$ congrès mondial de Linguistique française - CMLF 2012 (Lyon, 4-7 Juillet 2012). Les Ulis: EDP Sciences. Vol. 1 : 2451-2469. En ligne à l'adresse suivante: https:// www.shs-conferences.org/articles/shsconf/pdf/2012/01/shsconf_cmlf12_000172.pdf.

Thuilier, J., Abeillé, A. et Crabbé, B. 2014. Ordering Preferences for Postverbal Complements in French. In H. Tyne, V. André, C. Benzitoun, A. Boulton et Y. Greub (éd.), French through Corpora: Ecological and Data-Driven Perspectives in French Language Studies. Newcastle-upon-Tyne: Cambridge Scholars Publishing: 77-102.

Thuilier, J., Grant, M., Crabbé, B. et Abeillé, A. à paraître. Word Order in French: The Role of Animacy. Glossa: A Journal of General Linguistics.

Weiner, E. J. et Labov, W. 1983. Constraints on the Agentless Passive. Journal of Linguistics 19 (1) : 29-58.

ZRIBI-HeRTZ, A. 1982. La construction “se-moyen" du français et son statut dans le triangle: moyen/passif/réfléchi. Lingvistice Investigationes 6 (2) : 345-401. 